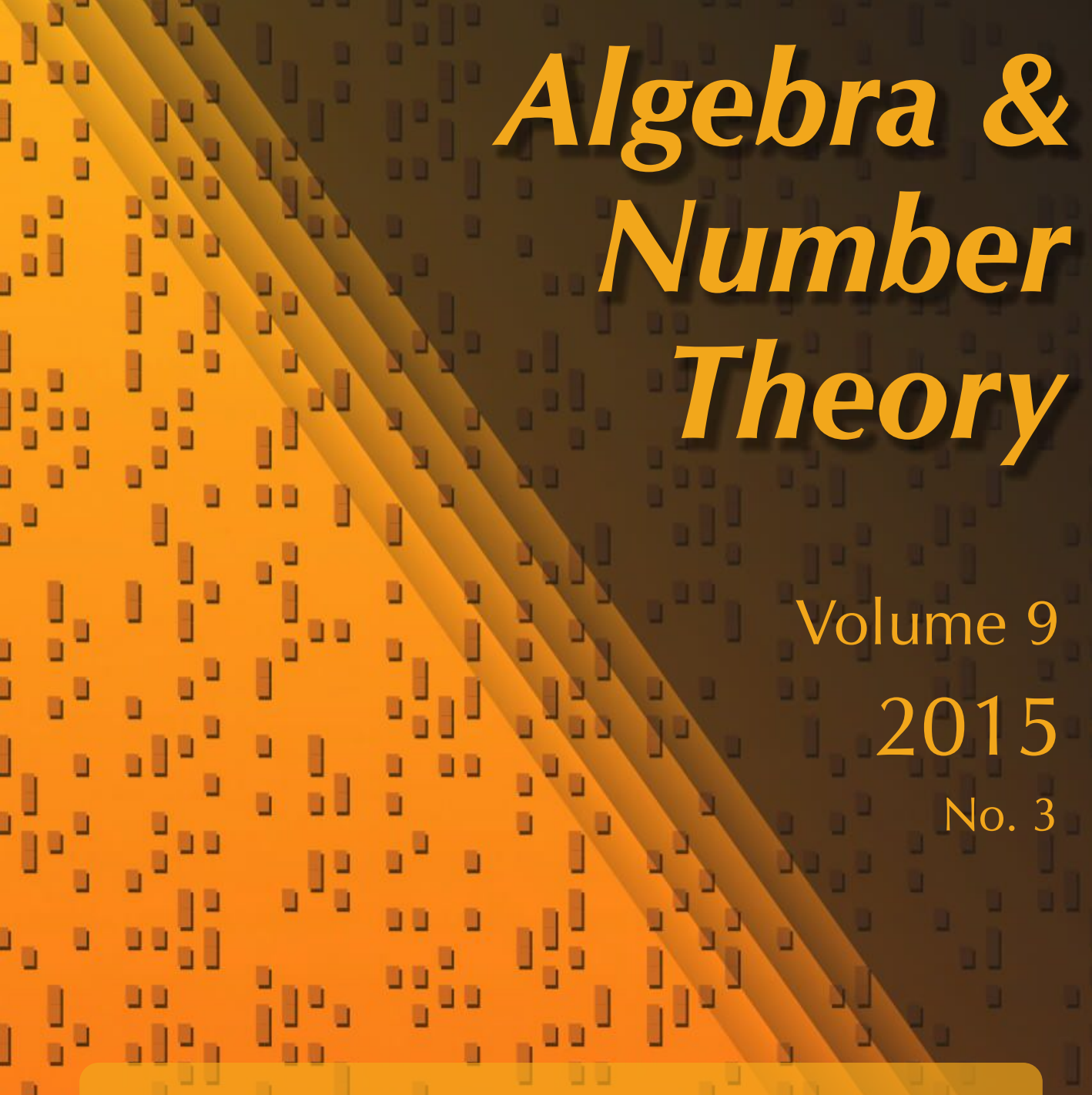

On the basepoint-free theorem for log canonical threefolds over the algebraic closure of a finite field

Diletta Martinelli, Yusuke Nakamura and Jakub Witaszek

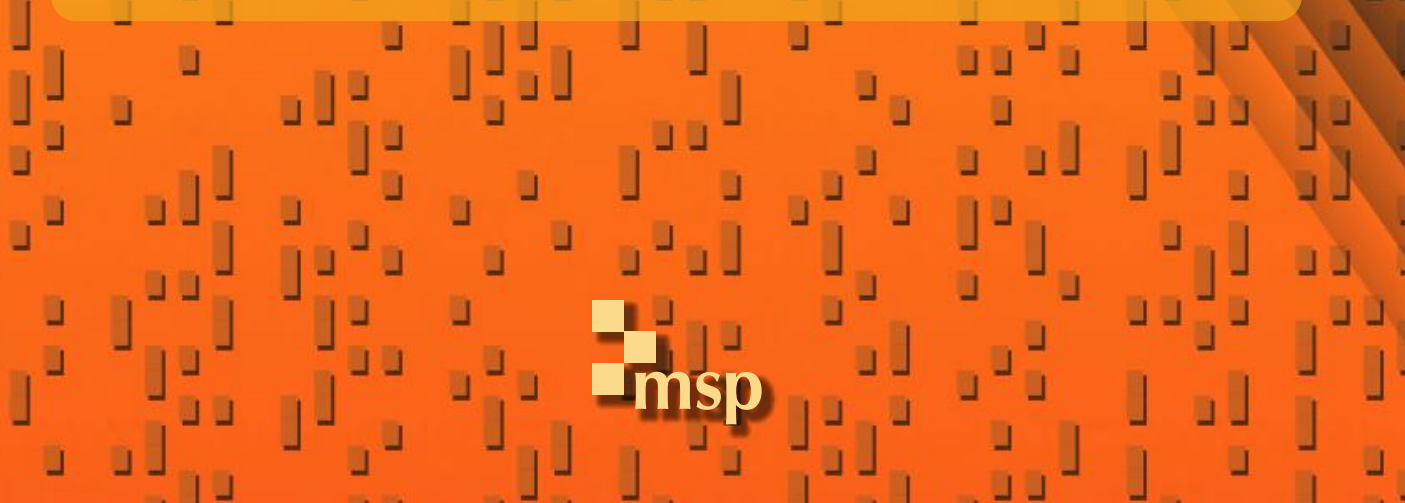




\title{
On the basepoint-free theorem for log canonical threefolds over the algebraic closure of a finite field
}

\author{
Diletta Martinelli, Yusuke Nakamura and Jakub Witaszek
}

We prove the basepoint-free theorem for big line bundles on a three-dimensional $\log$ canonical projective pair defined over the algebraic closure of a finite field. This theorem is not valid for any other algebraically closed field.

\section{Introduction}

A line bundle $L$ is called semiample if some positive tensor power $L^{\otimes r}$ is generated by global sections. Semiample line bundles play an important role in algebraic geometry, because they determine morphisms of a variety into projective spaces. Therefore, one would like to find necessary and sufficient conditions for semiampleness. A semiample line bundle is necessarily nef, but the converse is false in general. However, if we assume that $L$ is the canonical bundle and is nef, then the abundance conjecture [Kollár and Mori 1998, Conjecture 3.12] states that $L$ must be semiample. Furthermore, the basepoint-free theorem [Kollár and Mori 1998, Theorem 3.3] asserts that a nef line bundle $L$ on a Kawamata $\log$ terminal projective pair $(X, \Delta)$ defined over an algebraically closed field of characteristic zero is semiample when $L-\left(K_{X}+\Delta\right)$ is nef and big.

In positive characteristic, questions regarding semiampleness are more difficult, due to the absence of a proof of the resolution of singularities for varieties of dimension greater than three and the failure of the Kawamata-Viehweg vanishing theorem. As such, the basepoint-free theorem remains still unsolved in general. However, many partial results for threefolds may be obtained by reductions to the two-dimensional cases.

The basepoint-free theorem in positive characteristic is known for big line bundles $L$ when $(X, \Delta)$ is a three-dimensional Kawamata log terminal projective pair defined over an algebraically closed field of characteristic larger than five (see [Birkar 2013; $\mathrm{Xu} 2013]$ ). Over $\overline{\mathbb{F}}_{p}$, the algebraic closure of a finite field, there is a stronger result,

MSC2010: primary 14E30; secondary 14C20.

Keywords: basepoint-free theorem, semiample line bundles, positive characteristic, finite fields. 
due to Keel [1999], who proved the basepoint-free theorem for big line bundles $L$ when $(X, \Delta)$ is a three-dimensional projective log pair defined over $\overline{\mathbb{F}}_{p}$ with all coefficients of $\Delta$ less than one.

In this paper, we generalize Keel's result to the cases where the coefficients of $\Delta$ may be equal to one. Our main theorem is the following:

Theorem 1.1. Let $(X, \Delta)$ be a three-dimensional projective log pair defined over $\overline{\mathbb{F}}_{p}$. Assume that one of the following conditions holds:

(1) $(X, \Delta)$ is log canonical.

(2) All the coefficients of $\Delta$ are at most one and each irreducible component of $\operatorname{Supp}(\lfloor\Delta\rfloor)$ is normal.

Let $L$ be a nef and big line bundle on X. If $L-\left(K_{X}+\Delta\right)$ is also nef and big, then $L$ is semiample.

The next corollary follows easily from Theorem 1.1.

Corollary 1.2. Let $(X, \Delta)$ be a three-dimensional log canonical projective pair defined over $\overline{\mathbb{F}}_{p}$.

(1) If $K_{X}+\Delta$ is nef and big, then $K_{X}+\Delta$ is semiample.

(2) If $-\left(K_{X}+\Delta\right)$ is nef and big, then $-\left(K_{X}+\Delta\right)$ is semiample.

Remark 1.3. Theorem 1.1 does not hold over fields $k \neq \overline{\mathbb{F}}_{p}$ even in the twodimensional case (Example 7.2). Corollary 1.2(2) also does not hold over algebraically closed fields $k \neq \overline{\mathbb{F}}_{p}$ (Example 7.3).

In Example 7.1, we give a counterexample to Theorem 1.1 if one does not impose any conditions on the effective $\mathbb{Q}$-divisor $\Delta$. It is not clear whether the theorem remains true if we only assume that all the coefficients of $\Delta$ are at most one.

We also prove the basepoint-free theorem for normal surfaces defined over $\overline{\mathbb{F}}_{p}$ without assuming bigness:

Theorem 1.4. Let $X$ be a normal projective surface defined over $\overline{\mathbb{F}}_{p}$ and let $\Delta$ be an effective $\mathbb{Q}$-divisor. Assume that we have a nef line bundle $L$ on $X$ such that $L-\left(K_{X}+\Delta\right)$ is also nef. Then $L$ is semiample.

Remark 1.5. It is not true in general that nef line bundles on smooth surfaces over $\overline{\mathbb{F}}_{p}$ are semiample (see Totaro's example [2009]).

Remark 1.6. Theorem 1.1 and Theorem 1.4 hold if we assume that $L$ is only a $\mathbb{Q}$-Cartier $\mathbb{Q}$-divisor. Note that if $L$ and $L-\left(K_{X}+\Delta\right)$ are big and nef, then

$$
n L-\left(K_{X}+\Delta\right)=(n-1) L+\left(L-\left(K_{X}+\Delta\right)\right)
$$

is also big and nef for any integer $n \geq 1$. 
The paper is organized as follows: in Section 2, we review some definitions and facts from minimal model theory and about the conductor scheme. Further, we list some results from [Keel 1999] and show lemmas necessary for the proof of the main theorem. In Section 3, we prove the basepoint-free theorem for surfaces under weaker assumptions (Theorem 1.4). In Section 4, generalizing the proof of [Keel 1999, Theorem 0.5], we reduce Theorem 1.1 to showing that the line bundle $\left.L\right|_{\text {Supp }\lfloor\Delta\rfloor}$ is semiample (Theorem 4.1). If $\operatorname{Supp}\lfloor\Delta\rfloor$ is irreducible, we know that $\left.L\right|_{\operatorname{Supp}\lfloor\Delta\rfloor}$ is semiample by Theorem 1.4. The nonirreducible case is treated in Section 5. In order to generalize Theorem 1.4 to the nonirreducible surfaces, we combine ideas from Fujino [2000] and Tanaka [2014], together with special properties of varieties defined over $\overline{\mathbb{F}}_{p}$, which are proved in Section 2. In Section 6, we complete the proof of Theorem 1.1 and of Corollary 1.2. In Section 7, we give the counterexamples stated in Remark 1.3.

Notation and conventions. - When we work over a normal variety $X$, we often identify a line bundle $L$ with the divisor corresponding to $L$. For example, we use the additive notation $L+A$ for a line bundle $L$ and a divisor $A$.

- Following the notation of [Keel 1999], for a morphism $f: X \rightarrow Y$, a line bundle $L$ on $Y$, and a section $s \in H^{0}(Y, L)$, we denote by $\left.L\right|_{X}$ and $\left.s\right|_{X}$ the pullbacks $f^{*} L$ and $f^{*} s$, respectively.

- With the same notation as above, we say that a section $t \in H^{0}\left(X,\left.L\right|_{X}\right)$ descends to $Y$ if there exists a section $s \in H^{0}(Y, L)$ such that $f^{*} s=t$.

- Let $X$ be a reduced scheme of finite type over a field, $X=\bigcup X_{i}$ the decomposition into irreducible components, and $\overline{X_{i}} \rightarrow X_{i}$ the normalizations. Then we define the normalization of $X$ as the composition $\bigsqcup \overline{X_{i}} \rightarrow \bigsqcup X_{i} \rightarrow X$.

- Let $X$ be a scheme and $F \subset X$ a closed subscheme. Let $L$ be a line bundle on $X$ and $s \in H^{0}(X, L)$ its section. We say that $s$ is nowhere-vanishing on $F$ if $\left.s\right|_{\{x\}}$ is not zero as an element in the one-dimensional vector space $H^{0}\left(\{x\},\left.L\right|_{\{x\}}\right)$ for any closed point $x \in F$.

- We say that a line bundle $L$ on $X$ is semiample when the linear system $|m L|$ is basepoint-free for a sufficiently large and divisible positive integer $m$. When $L$ is semiample, the surjective map $f: X \rightarrow Y$ defined by $|m L|$ satisfies $f_{*} \mathscr{O}_{X}=\mathscr{O}_{Y}$ for a sufficiently large and divisible positive integer $m$. We call $f$ the map associated to $L$.

\section{Preliminaries}

2A. Log pairs. A log pair $(X, \Delta)$ is a normal variety $X$ and an effective $\mathbb{Q}$-divisor $\Delta$ such that $K_{X}+\Delta$ is $\mathbb{Q}$-Cartier. 
For a proper birational morphism $f: X^{\prime} \rightarrow X$ from a normal variety $X^{\prime}$, we write

$$
K_{X^{\prime}}+\sum_{i} a_{i} E_{i}=f^{*}\left(K_{X}+\Delta\right)
$$

where the $E_{i}$ are prime divisors. We say that the pair $(X, \Delta)$ is $\log$ canonical if $a_{i} \leq 1$ for any proper birational morphism $f$. Further, we say that the pair $(X, \Delta)$ is Kawamata log terminal if $a_{i}<1$ for any proper birational morphism $f$.

2B. Conductor schemes. Let $X$ be a reduced scheme of finite type over a field and $\bar{X} \rightarrow X$ its normalization. We identify the sheaf of rings $\mathscr{O}_{X}$ as a subring of $0_{\bar{X}}$. Let $\mathscr{I} \subset O_{X}$ be the maximal ideal sheaf satisfying $\mathscr{I} \mathscr{O}_{\bar{X}} \subset O_{X}$. The conductor of $X$ is the subscheme $\mathscr{D} \subset X$ defined by $\mathscr{I}$. By abuse of notation, the subscheme $\mathscr{C} \subset \bar{X}$ defined by $\mathscr{T} \mathscr{O}_{\bar{X}}$ will also be called the conductor.

The notion of conductor is important to descend sections, because of the following remark:

Remark 2.1. Let $\mathscr{b} \subset \bar{X}, \mathscr{D} \subset X$ be conductors and let $L$ be a line bundle on $X$ :

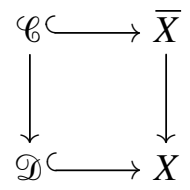

By definition of the conductor, we have the following exact sequence

$$
0 \longrightarrow H^{0}(X, L) \longrightarrow H^{0}\left(\bar{X},\left.L\right|_{\bar{X}}\right) \oplus H^{0}\left(\mathscr{D},\left.L\right|_{\mathscr{D}}\right) \longrightarrow H^{0}\left(\mathscr{C},\left.L\right|_{\mathscr{C}}\right),
$$

where the second map is defined by $t \mapsto\left(\left.t\right|_{\bar{X}},\left.t\right|_{\mathscr{D}}\right)$ and the third map is defined by $\left.(t, u) \mapsto t\right|_{\mathscr{C}}-\left.u\right|_{\mathscr{C}}$. Therefore, a section $s \in H^{0}\left(\bar{X},\left.L\right|_{\bar{X}}\right)$ descends to $X$ if and only if $\left.s\right|_{\mathscr{C}}$ descends to $\mathscr{D}$.

2C. Adjunction formula. Let $(X, \Delta)$ be a log pair and $S$ the union of the supports of some of the divisors with coefficient one in $\Delta$. Let $p: \bar{S} \rightarrow S$ be the normalization of $S$. Then there exists an effective $\mathbb{Q}$-divisor $\Delta_{\bar{S}}$ on $\bar{S}$ such that

$$
K_{\bar{S}}+\Delta_{\bar{S}}=\left.\left(K_{X}+\Delta\right)\right|_{\bar{S}}
$$

holds (see for instance [Kollár 2013, Definition 4.2]).

We denote by $C$ the possibly nonreduced divisor on $\bar{S}$ corresponding to the codimension-one part of $\mathscr{C}$, where $\mathscr{C} \subset \bar{S}$ is the conductor of $S$.

When $X$ is $\mathbb{Q}$-factorial, it follows that $C \leq \Delta_{\bar{S}}$ by [Keel 1999, Theorem 5.3]. In this paper, we use the following proposition, which only states $\operatorname{Supp}(C) \subset$ $\operatorname{Supp}\left(\left\lfloor\Delta_{\bar{S}}\right\rfloor\right)$, but is valid even for a non- $\mathbb{Q}$-factorial variety $X$. 
Proposition 2.2. Let $(X, \Delta)$ be a log pair, and let $S$ be the union of the supports of some of the divisors with coefficient one in $\Delta$. Let $p: \bar{S} \rightarrow S$ be the normalization of $S$, and let $\Delta_{\bar{S}}$ be an effective $\mathbb{Q}$-divisor on $\bar{S}$ defined by the adjunction as above. Further, we denote by $C$ the (possibly nonreduced) divisor on $\bar{S}$ corresponding to the codimension-one part of $\mathscr{C}$, where $\mathscr{b} \subset \bar{S}$ is the conductor of $S$. Then the following hold:

(1) $\operatorname{Supp}(C) \subset \operatorname{Supp}\left(\left\lfloor\Delta_{\bar{S}}\right\rfloor\right)$.

(2) Let $D_{1}, \ldots, D_{c}$ be prime divisors with coefficient greater than or equal to one in $\Delta$, and let $T=\bigcup_{1 \leq i \leq c} \operatorname{Supp}\left(D_{i}\right)$. Assume that each $D_{i}$ satisfies $\operatorname{Supp}\left(D_{i}\right) \not \subset S$. Then, the codimension-one part of $p^{-1}(S \cap T)$ is contained in $\operatorname{Supp}\left(\left\lfloor\Delta_{\bar{S}}\right\rfloor\right)$.

Proof. First we prove (1). Let $V \subset \bar{S}$ be a codimension-one subvariety such that $V \subset \mathscr{C}$. It is sufficient to show $\operatorname{coeff}_{V} \Delta_{\bar{S}} \geq 1$. When $(X, \Delta)$ is not log canonical at the generic point $\eta_{p(V)}$ of $p(V)$, we have coeff $\Delta_{\bar{S}}>1$ (see [Kollár 2013, Proposition 4.5(2)]). Hence, we may assume that $(X, \Delta)$ is log canonical at $\eta_{p(V)}$. In this case, $S$ has a node at $\eta_{p(V)}$ and $\operatorname{coeff}_{V} \Delta_{\bar{S}}=1$ (see the proof of [Kollár 2013, Proposition 4.5(6)]).

Next, we prove (2). Let $V \subset \bar{S}$ be a codimension-one subvariety such that $V \subset p^{-1}(S \cap T)$. It is sufficient to show $\operatorname{coeff}_{V} \Delta_{\bar{S}} \geq 1$. Since the problem is local around $V$, we may assume that $p(V) \subset \operatorname{Supp}\left(D_{i}\right)$ for all $i$. If coeff $D_{D_{i}} \Delta>1$ for some $i$, then $(X, \Delta)$ is not $\log$ canonical at the generic point $\eta_{p(V)}$ of $p(V)$. In this case, we have coeff ${ }_{V} \Delta_{\bar{S}}>1$ as above. Hence, we may assume that coeff $D_{D_{i}} \Delta=1$ for all $i$. Note that $S \cap T$ is contained in the conductor of the normalization of $S \cup T$. Therefore, we conclude the proof by applying (1) to $S \cup T$.

2D. Some properties of varieties over $\overline{\mathbb{F}}_{p}$. The following fact distinguishes $\overline{\mathbb{F}}_{p}$ from other fields of positive characteristic. For the proof, see for instance [Keel 1999, Lemma 2.16].

Proposition 2.3. The Picard scheme $\mathrm{Pic}^{0} X$ is a torsion group when $X$ is a projective scheme defined over $\overline{\mathbb{F}}_{p}$. In particular, any numerically trivial Cartier divisor is $\mathbb{Q}$-linearly trivial.

We need the following lemmas in Section 5:

Lemma 2.4. Let $X$ be a proper scheme over $\overline{\mathbb{F}}_{p}$. Let $s_{1}, s_{2} \in H^{0}\left(X, O_{X}\right)$ be sections of the structure sheaf. Assume that $s_{1}$ and $s_{2}$ are nowhere-vanishing on $X$. Then there exists $n \geq 1$ such that $s_{1}^{n}=s_{2}^{n}$ in $H^{0}\left(X, O_{X}\right)$.

Proof. Without loss of generality we may assume that $X$ is connected. Set $A:=$ $H^{0}\left(X, O_{X}\right)$. It is a finite-dimensional vector space over $\overline{\mathbb{F}}_{p}$, because $X$ is proper. 
Since $X$ is connected, $A$ has a unique maximal ideal $\mathfrak{m}$, and it follows that $A / \mathfrak{m} \cong$ $H^{0}\left(X^{\mathrm{red}}, \mathrm{O}_{X^{\mathrm{red}}}\right) \cong \overline{\mathbb{F}}_{p}$.

Let $a_{i}$ be the element of $A$ corresponding to $s_{i}$, and $\overline{a_{i}}$ the image of $a_{i}$ in $\overline{\mathbb{F}}_{p}$. Since $s_{i}$ is nowhere-vanishing on $X$, the element $\overline{a_{i}} \in \overline{\mathbb{F}}_{p}$ is not zero. Hence, there exists $e \geq 1$ for which $\overline{a_{1}} p^{e}-1=\overline{a_{2}} p^{e}-1=1$. Take $r \geq 1$ such that $\mathfrak{m}^{p^{r}}=0$. Then we have

$$
a_{1}^{p^{r}\left(p^{e}-1\right)}-a_{2}^{p^{r}\left(p^{e}-1\right)}=\left(a_{1}^{p^{e}-1}-a_{2}^{p^{e}-1}\right)^{p^{r}} \in \mathfrak{m}^{p^{r}}=0 .
$$

Therefore, it is sufficient to set $n=p^{r}\left(p^{e}-1\right)$.

Lemma 2.5. Let $X$ be a one-dimensional reduced scheme of finite type over $\overline{\mathbb{F}}_{p}$, $L$ a line bundle on $X$, and $p: \bar{X} \rightarrow X$ the normalization of $X$. Let $\mathscr{b} \subset \bar{X}$ be the conductor of $X$, and $s \in H^{0}\left(\bar{X},\left.L\right|_{\bar{X}}\right)$ a section nowhere-vanishing on $\mathscr{C}$. Then $s^{n}$ descends to $X$ for some $n \geq 1$.

Proof. Let $\mathscr{D} \subset X$ be the conductor. Note that $\mathscr{C}$ and $\mathscr{D}$ are either empty or have dimension zero. By Remark 2.1, it is sufficient to prove that $\left.s^{n}\right|_{\mathscr{C}}$ descends to $\mathscr{D}$ for some $n \geq 1$. Let $t \in H^{0}\left(\mathscr{D},\left.L\right|_{\mathscr{D}}\right)$ be a section nowhere-vanishing on $\mathscr{D}$. Then $\left.t\right|_{\mathscr{C}}$ is nowhere-vanishing on $\mathscr{C}$. Any line bundle is trivial on a zero-dimensional scheme, and so, by Lemma 2.4, we get $\left.s^{n}\right|_{\mathscr{C}}=\left.t^{n}\right|_{\mathscr{C}}$ for some $n \geq 1$. In particular, $\left.s^{n}\right|_{\mathscr{C}}$ descends to $\mathscr{D}$.

Lemma 2.6. Let $C$ be a smooth proper connected curve over $\overline{\mathbb{F}}_{p}$. Then a finitely generated subgroup of $\operatorname{Aut}(C)$ is finite.

Proof. If $g(C) \geq 2$, then $\operatorname{Aut}(C)$ is finite and the statement is trivial. If $C=\mathbb{P}^{1}$, then $\operatorname{Aut}(C) \cong \operatorname{PGL}\left(2, \overline{\mathbb{F}}_{p}\right)$. A finitely generated subgroup $G$ of $\operatorname{PGL}\left(2, \overline{\mathbb{F}}_{p}\right)$ is always finite, because $G$ is contained in $\operatorname{PGL}\left(2, \mathbb{F}_{p^{e}}\right)$ for some $e \geq 1$. If $C$ is an elliptic curve, then we get $\operatorname{Aut}(C) \cong T \rtimes F$, where $T$ is the group of translations and $F$ is a finite group (see for instance [Silverman 2009, Section X.5]). Note that each element of $T$ has finite order, because $C$ is defined over $\overline{\mathbb{F}}_{p}$. Hence, a finitely generated subgroup of the abelian group $T$ is always finite, and so a finitely generated subgroup of $\operatorname{Aut}(C)$ is also finite.

For completeness, we note a general fact in group theory: any finitely generated subgroup of $G_{1} \rtimes G_{2}$ is finite, if we assume that any finitely generated subgroup of $G_{i}$ is finite for each $i$.

2E. Keel's theorems. The following theorem is crucial in reducing problems from threefolds to surfaces:

Theorem 2.7 [Keel 1999, Proposition 1.6]. Let X be a projective scheme over a field of positive characteristic. Let $L$ be a nef line bundle on $X$, and let $E$ be an effective Cartier divisor on $X$ such that $L-E$ is ample. Then $L$ is semiample if and only if $\left.L\right|_{E_{\text {red }}}$ is semiample. 
We note that Cascini, $M^{c}$ Kernan, and Mustață [Cascini et al. 2014, Theorem 3.2] gave a different proof of Theorem 2.7.

Theorem 2.8 [Artin 1962, Theorem 2.9; Keel 1999, Corollary 0.3]. Let X be a projective surface over $\overline{\mathbb{F}}_{p}$, and let $L$ be a nef and big line bundle on $X$. Then $L$ is semiample.

Proof. Since by Proposition 2.3 nef line bundles on curves over $\overline{\mathbb{F}}_{p}$ are semiample, the claim follows from Theorem 2.7.

We say that a map $f: X \rightarrow Y$ is a finite universal homeomorphism if it is a finite homeomorphism under any base change. In this case, we have a correspondence, up to taking powers, between the set of sections of a line bundle $L$ on $Y$ and the set of sections of $\left.L\right|_{X}$.

Theorem 2.9 [Keel 1999, Lemma 1.4]. Let $f: X \rightarrow Y$ be a finite universal homeomorphism between varieties defined over a field of characteristic $p>0$, and let $L$ be a line bundle on $Y$. Then the following hold:

(1) For $s \in H^{0}\left(X,\left.L\right|_{X}\right)$, the section $s^{p^{e}} \in H^{0}\left(X,\left.L^{\otimes p^{e}}\right|_{X}\right)$ descends to $Y$ for a sufficiently large integer $e \geq 1$.

(2) If $t \in H^{0}(Y, L)$ satisfies $\left.t\right|_{X}=0$, then $t^{p^{e}}=0$ holds for a sufficiently large integer $e \geq 1$.

In this paper, we frequently use the following theorems:

Theorem 2.10 [Keel 1999, Corollary 2.12]. Let $X=X_{1} \cup X_{2}$ be a projective scheme over $\overline{\mathbb{F}}_{p}$, where the $X_{i}$ are closed subsets. Let $L$ be a nef line bundle on $X$ such that the $\left.L\right|_{X_{i}}$ are semiample. Let $g_{i}: X_{i} \rightarrow Z_{i}$ be the map associated to $\left.L\right|_{X_{i}}$. Assume that all but finitely many fibers of $\left.g_{2}\right|_{X_{1} \cap X_{2}}$ are geometrically connected. Then $L$ is semiample.

Theorem 2.11 [Keel 1999, Corollary 2.14]. Let X be a reduced projective scheme over $\overline{\mathbb{F}}_{p}$. Let $p: \bar{X} \rightarrow X$ be the normalization of $X$. Let $D \subset X$ and $C \subset \bar{X}$ be the reductions of the conductors. Let $L$ be a nef line bundle on $X$ such that $\left.L\right|_{\bar{X}}$ and $\left.L\right|_{D}$ are semiample. Let $g: \bar{X} \rightarrow Z$ be the map associated to $\left.L\right|_{\bar{X}}$. Assume that all but finitely many fibers of $\left.g\right|_{C}$ are geometrically connected. Then $L$ is semiample.

\section{Basepoint-free theorem for normal surfaces}

In this section, we prove Theorem 1.4. The key tool is the following theorem of Tanaka. We say that a $\mathbb{Q}$-divisor $B$ on a variety $X$ is $\mathbb{Q}$-effective if $h^{0}(X, m B) \neq 0$ for some $m \geq 1$. Note that a normal surface over $\overline{\mathbb{F}}_{p}$ is always $\mathbb{Q}$-factorial (see [Tanaka 2012, Theorem 11.1]). 
Theorem 3.1 [Tanaka 2012, Theorem 12.6]. Let X be a projective normal surface over $\overline{\mathbb{F}}_{p}$ and let $D$ be a nef divisor. If $q D-K_{X}$ is $\mathbb{Q}$-effective for some positive rational number $q \in \mathbb{Q}$, then $D$ is semiample.

We will use the following proposition to reduce the case of hyperelliptic surfaces to abelian surfaces:

Proposition 3.2. Let $p: Y \rightarrow X$ be a proper surjection between varieties defined over an algebraically closed field, and let $L$ be a line bundle on $X$. Assume that $X$ is normal. Then $L$ is semiample if and only if $p^{*}(L)$ is semiample.

Proof. See for instance the proof of [Keel 1999, Lemma 2.10].

Proof of Theorem 1.4. Recall that we have the nef line bundle $L$ and the $\mathbb{Q}$-divisor $D:=L-\left(K_{X}+\Delta\right)$ on the normal surface $X$ over $\overline{\mathbb{F}}_{p}$.

Claim 3.3. We can assume that $X$ is smooth.

Proof. Let $f: Y \rightarrow X$ be the minimal resolution of singularities of $X$. Define $\Delta_{Y}$ so that $K_{Y}+\Delta_{Y}=f^{*}\left(K_{X}+\Delta\right)$. The divisor $\Delta_{Y}$ is an effective $\mathbb{Q}$-divisor by the negativity lemma (see [Kollár and Mori 1998, Corollary 4.3]). Note that $f^{*} L$ and $f^{*} D=f^{*} L-\left(K_{Y}+\Delta_{Y}\right)$ are nef. By Proposition 3.2 we know that $L$ is semiample if and only if $f^{*} L$ is semiample. Thus, by replacing $X$ by $Y$, we may assume that the surface is smooth.

We extensively use the following lemma:

Lemma 3.4. If $D$ is $\mathbb{Q}$-effective, then $L$ is semiample.

Proof. Since $D$ is $\mathbb{Q}$-effective, $L-K_{X}=D+\Delta$ is also $\mathbb{Q}$-effective, and so $L$ is semiample by Theorem 3.1.

Claim 3.5. We can assume that all the following statements are true.
(1) $L \not \equiv 0$ and $D \not \equiv 0$,
(2) $L^{2}=0$,
(3) $D^{2}=0$,
(4) $L \cdot \Delta=0$,
(5) $L \cdot K_{X}=0$,
(6) $\left(K_{X}+\Delta\right) \cdot \Delta=0$,
(7) $\left(K_{X}+\Delta\right) \cdot K_{X}=0$,
(8) $\chi\left(\mathbb{O}_{X}\right) \leq 0$.

Proof. If $L \equiv 0$, then $L \sim_{\mathbb{Q}} O_{X}$ by Proposition 2.3, so $L$ is semiample. Thus, we may assume that $L \not \equiv 0$. Analogously, we may assume that $D \not \equiv 0$.

As $L$ and $D$ are nef, we get $L^{2} \geq 0$ and $D^{2} \geq 0$. If $L^{2}>0$, then, by Theorem 2.8, the line bundle $L$ is semiample. Thus, we may assume that $L^{2}=0$. If $D^{2}>0$, then $D$ is big, and so $\mathbb{Q}$-effective. In this case $L$ is semiample by Lemma 3.4. Hence, we may assume $D^{2}=0$.

Since $L \not \equiv 0$, we know that there exists a curve $C$ on $X$ satisfying $L \cdot C>0$. Take an ample divisor $A$ such that $A-C$ is effective. Then $L \cdot A=L \cdot C+L \cdot(A-C)>0$. 
If $m$ is sufficiently large that it satisfies $\left(K_{X}-m L\right) \cdot A<0$, then $h^{2}(X, m L)=$ $h^{0}\left(X, K_{X}-m L\right)=0$. The Riemann-Roch theorem gives

$$
\begin{aligned}
h^{0}(X, m L) & =h^{1}(X, m L)+\frac{1}{2} m L \cdot\left(m L-K_{X}\right)+\chi\left(\mathscr{O}_{X}\right) \\
& =h^{1}(X, m L)-\frac{1}{2} m L \cdot K_{X}+\chi\left(\mathscr{O}_{X}\right) .
\end{aligned}
$$

As $L$ and $D$ are nef, it follows that

$$
0 \leq L \cdot D=-L \cdot K_{X}-L \cdot \Delta .
$$

Since $\Delta$ is effective and $L$ is nef, we find $0 \leq L \cdot D \leq-L \cdot K_{X}$. If $-L \cdot K_{X}>0$, then $\kappa(X, L)=1$ by the calculation of $h^{0}(X, m L)$ above. A nef line bundle $L$ with $\kappa(X, L)=1$ is always semiample (see for instance [Fong and $\mathrm{M}^{\mathrm{c}}$ Kernan 1992, Theorem 11.3.1]). Thus, we may assume that $L \cdot \Delta=0$ and $L \cdot K_{X}=0$.

As above, $h^{2}(X, m D)=0$ holds for sufficiently large $m$, and so the RiemannRoch theorem gives

$$
\begin{aligned}
h^{0}(X, m D) & =h^{1}(X, m D)-\frac{1}{2} m D \cdot K_{X}+\chi\left(\mathbb{O}_{X}\right) \\
& =h^{1}(X, m D)+\frac{1}{2} m D \cdot(D-L+\Delta)+\chi\left(\mathbb{O}_{X}\right) \\
& =h^{1}(X, m D)+\frac{1}{2} m D \cdot \Delta+\chi\left(\mathbb{O}_{X}\right) \\
& =h^{1}(X, m D)-\frac{1}{2} m\left(K_{X}+\Delta\right) \cdot \Delta+\chi\left(\mathbb{O}_{X}\right) .
\end{aligned}
$$

If $-\left(K_{X}+\Delta\right) \cdot \Delta>0$, then $D$ is $\mathbb{Q}$-effective and by Lemma 3.4 the line bundle $L$ is semiample. Since $0 \leq D \cdot \Delta=-\left(K_{X}+\Delta\right) \cdot \Delta$ holds by the nefness of $D$, we may assume $\left(K_{X}+\Delta\right) \cdot \Delta=0$. Given $D^{2}=L^{2}=D \cdot L=0$, it follows that $\left(K_{X}+\Delta\right) \cdot K_{X}=0$.

By the Riemann-Roch theorem, we get $h^{0}(X, m D)=h^{1}(X, m D)+\chi\left(\mathbb{O}_{X}\right)$. If $\chi\left(O_{X}\right)>0$, then $D$ is $\mathbb{Q}$-effective and by Lemma 3.4 the line bundle $L$ is semiample. Hence, we may assume that $\chi\left(O_{X}\right) \leq 0$.

We divide the proof into cases depending on the Kodaira dimension.

Case 1: Assume $\kappa(X) \geq 0$.

Claim 3.6. We may assume that $K_{X}$ is nef.

Proof. Let $\pi: X \rightarrow X_{\min }$ be the minimal model of $X$. By $\pi_{*} L$ we denote the pushforward of $L$ as a divisor.

By the assumption $\kappa(X) \geq 0$, we have that $K_{X}$ is $\mathbb{Q}$-linearly equivalent to an effective $\mathbb{Q}$-divisor containing every $\pi$-exceptional curve in its support. Since $L \cdot K_{X}=0$ and $L$ is nef, it follows that $L \cdot E=0$ for every $\pi$-exceptional curve $E$. Hence, we get $L=\pi^{*} \pi_{*} L$ by the negativity of the intersection form on the exceptional locus (see [Kollár and Mori 1998, Lemma 3.40]). 
Since $L=\pi^{*} \pi_{*} L$, it is sufficient to show the semiampleness of $\pi_{*} L$. Note that $\pi_{*} L$ and $\pi_{*} D$ are nef, because $L$ and $D$ are nef. Further, we have $\pi_{*} D=$ $\pi_{*} L-\left(K_{X_{\min }}+\pi_{*} \Delta\right)$. Therefore, we can reduce the problem to the case of the minimal model $X_{\min }$.

In what follows, we assume that $X$ is minimal. We use the classification of minimal surfaces in positive characteristic (see for instance [Mumford 1969; Bombieri and Mumford 1977; 1976; Liedtke 2013]).

Case 1.1: Assume $\kappa(X)=2$.

We can write $K_{X}=A+E$ for an ample $\mathbb{Q}$-divisor $A$ and an effective $\mathbb{Q}$-divisor $E$, because $K_{X}$ is big. Since $L, D$ are nef and $L \cdot K_{X}=D \cdot K_{X}=0$, it follows that $L \cdot A=D \cdot A=0$. Thus, $(L-D) \cdot A=\left(K_{X}+\Delta\right) \cdot A=0$. We get a contradiction

$$
0<A^{2} \leq\left(K_{X}+\Delta\right) \cdot A=0 .
$$

Hence, there are no line bundles $L$ satisfying the assumptions in Claim 3.5.

Case 1.2: Assume $\kappa(X)=1$.

In our case, $K_{X}$ is semiample and it gives an elliptic or quasielliptic fibration $f: X \rightarrow B$. Let $F$ be its general fiber. Then $K_{X} \equiv a F$ holds for some positive rational number $a$.

Since $D \cdot K_{X}=0$, it follows that $D \cdot F=0$. Therefore, $D$ is $f$-numerically trivial by the nefness of $D$. Since $D$ is nef and $f$-numerically trivial, it satisfies $D \equiv b F$ for some $b \geq 0$, by Lemma 3.7. Hence, $D$ is $\mathbb{Q}$-effective by Proposition 2.3. Therefore, $L$ is semiample by Lemma 3.4.

Lemma 3.7. Let $f: X \rightarrow B$ be a surjective morphism satisfying $f_{*}\left(\mathscr{O}_{X}\right)=\mathscr{O}_{B}$ from a smooth projective surface $X$ to a smooth projective curve $B$. Suppose that $L$ is an $f$-numerically trivial nef $\mathbb{Q}$-Cartier $\mathbb{Q}$-divisor. Then $L \equiv b F$ for some $b \geq 0$, where $F$ denotes a general fiber of $f$.

Proof. See for instance [Lehmann 2012, Lemma 2.4].

Case 1.3: Assume $\kappa(X)=0$.

By the classification of minimal surfaces, there are five possibilities: a K3 surface, an Enriques surface, an abelian surface, a hyperelliptic surface, or a quasihyperelliptic surface.

If $X$ is a $\mathrm{K} 3$ surface or an Enriques surface, then $\chi\left(0_{X}\right)=2$ or $\chi\left(0_{X}\right)=1$, respectively, which contradicts Claim 3.5.

If $X$ is an abelian surface, then every nef divisor is numerically equivalent to a semiample divisor (see Proposition 3.10). Therefore, $L$ is semiample by Proposition 2.3. 
If $X$ is a hyperelliptic surface, then $X$ is a finite quotient of an abelian surface by a finite group. Therefore, we have a surjective morphism $A \rightarrow X$ from an abelian surface $A$. Since $\left.L\right|_{A}$ is a nef line bundle on an abelian surface, it is semiample (see Proposition 3.10). Hence, $L$ is also semiample by Proposition 3.2.

If $X$ is a quasihyperelliptic surface, then $X$ can be written as a finite quotient $E \times C \rightarrow X$, where $E$ is an elliptic curve and $C$ is a rational curve with a cusp. Therefore, we have a surjective morphism $X^{\prime}:=E \times \mathbb{P}^{1} \rightarrow X$. Any divisor on $X^{\prime}$ is numerically equivalent to $a F_{1}+b F_{2}$ with $a, b \in \mathbb{Q}$, where $F_{1}$ is the fiber class of $X^{\prime} \rightarrow E$ and $F_{2}$ is the fiber class of $X^{\prime} \rightarrow \mathbb{P}^{1}$. Hence, any nef divisor on $X^{\prime}$ is numerically equivalent to a semiample divisor. Thus, we can conclude that $L$ is semiample by Proposition 2.3 and Proposition 3.2.

Case 2: Assume $\kappa(X)=-\infty$.

Since $\chi\left(O_{X}\right) \leq 0$, the surface $X$ is irrational. Thus, we can assume that $f: X \rightarrow B$ is a birationally ruled surface, where $B$ is a curve with $g(B) \geq 1$.

We need the following lemma, which can be found in the proof of [Tanaka 2012, Theorem 12.4].

Lemma 3.8. Let $C$ be an $f$-horizontal curve on $X$ such that $D \cdot C=0$. Then $D$ is $\mathbb{Q}$-effective.

Proof. Since $C$ is a horizontal curve, it holds that $g(B) \leq h^{1}\left(C, O_{C}\right)$. By the Riemann-Roch theorem, we get

$$
h^{0}(X, m D)=h^{1}(X, m D)+\chi\left(\mathcal{O}_{X}\right)=h^{1}(X, m D)+1-g(B),
$$

so it is sufficient to show $h^{1}(X, m D) \geq h^{1}\left(C, \mathscr{O}_{C}\right)$ for some $m>0$.

Since $D \cdot C=0$, we have $\left.D\right|_{C} \equiv 0$. Hence, by Proposition 2.3 we can conclude that $\left.m D\right|_{C}$ is trivial for a sufficiently divisible $m>0$. Therefore, we get an exact sequence

$$
0 \longrightarrow \mathrm{O}_{X}(m D-C) \longrightarrow \mathrm{O}_{X}(m D) \longrightarrow \mathrm{O}_{C} \longrightarrow 0
$$

By the same reason as before, $h^{2}(X, m D-C)=0$ holds for sufficiently large $m$. Hence, we get $h^{1}(X, m D) \geq h^{1}\left(C, O_{C}\right)$.

For any irreducible component $C$ of $\Delta$, it follows that $D \cdot C=0$, because $D$ is nef and $D \cdot \Delta=0$. In particular, if $\Delta$ has an $f$-horizontal component, then the lemma above implies that $D$ is $\mathbb{Q}$-effective, and hence $L$ is semiample by Lemma 3.4. Thus, in what follows, we may assume that $\Delta$ has only $f$-vertical components.

Claim 3.9. Under these assumptions, it follows that $\Delta=0, g(B)=1$, and $X$ is a minimal ruled surface. 
Proof. Let $\pi: X \rightarrow X_{\min }$ be a minimal model of $X$. We have $K_{X} \sim \pi^{*} K_{X_{\min }}+E$, where $E$ is an exceptional divisor. We refer the reader to [Hartshorne 1977, Chapter V, Section 2] for properties of ruled surfaces. It holds that

$$
K_{X_{\min }} \equiv-2 C_{0}+(2 g(B)-2-e) F
$$

for $C_{0}$ a normalized section, $e=-C_{0}^{2}$, and $F$ a general fiber of $X_{\min } \rightarrow B$. Note that $K_{X_{\min }}^{2}=8(1-g(B))$.

Since $\left(K_{X}+\Delta\right) \cdot \Delta=0$ and $\left(K_{X}+\Delta\right) \cdot K_{X}=0$, we get

$$
\Delta^{2}=-K_{X} \cdot \Delta=K_{X}^{2} \text {. }
$$

As $\Delta$ has only $f$-vertical components, we have $\pi^{*} F \cdot \Delta=0$, and so

$$
0=\left(K_{X}+\Delta\right) \cdot \Delta=-2 \pi^{*} C_{0} \cdot \Delta+(E+\Delta) \cdot \Delta .
$$

Since $\pi^{*} C_{0} \cdot \Delta \geq 0$, it follows that $E \cdot \Delta \geq-\Delta^{2}$. Therefore,

$(E+\Delta)^{2}=E^{2}+2 E \cdot \Delta+\Delta^{2} \geq E^{2}-\Delta^{2}=E^{2}-K_{X}^{2}=-K_{X_{\min }}^{2}=8(g(B)-1) \geq 0$.

By the Zariski lemma [Liu 2002, Section 9, Theorem 1.23], the intersection form on $f$-vertical fibers is seminegative-definite with one-dimensional radical equal to the span of a general fiber, so $(E+\Delta)^{2}=0$ and $E+\Delta \equiv \pi^{*} p F$ for some $p \in \mathbb{Q}$.

Since all the inequalities must be equalities, it follows that $E \cdot \Delta=-\Delta^{2}$ and $g(B)=1$. Furthermore, we have $2 \pi^{*} C_{0} \cdot \Delta=(E+\Delta) \cdot \Delta$, and thus

$$
0=\pi^{*} C_{0} \cdot \Delta=\pi^{*} C_{0} \cdot(E+\Delta)=p .
$$

This implies that $E+\Delta=0$. Since $\Delta$ and $E$ are both effective divisors, we get $\Delta=0$ and $E=0$. Hence, $X$ is minimal.

By this claim, we can assume that $X$ is a minimal ruled surface over an elliptic curve. In this case, it is well-known that $\mathrm{NEF}(X) \subset \mathrm{NE}(X)$ holds (see Proposition 3.13). We can conclude that the nef divisor $D$ is $\mathbb{Q}$-effective and $L$ is semiample by Lemma 3.4.

For completeness, we prove two propositions which were used in the above proof: Proposition 3.10. Let $A$ be an abelian variety defined over an algebraically closed field. Then any nef line bundle on A is numerically equivalent to a semiample line bundle.

Remark 3.11. Note that any effective divisor on an abelian variety is always semiample (see the proof of Application $1(($ i $) \Rightarrow$ (iii)) in [Mumford 2008, Section 6]). Proof. Let $L$ be a nef line bundle on $A$. Define $K(L)$ to be the maximal subscheme of $A$ such that

$$
\left.\left(m^{*} L-p_{1}^{*} L-p_{2}^{*} L\right)\right|_{K(L) \times A}=\mathrm{O}_{K(L) \times A}
$$


as in [Mumford 2008, Section 13], where $m: A \times A \rightarrow A$ is the multiplication map and $p_{1}$ and $p_{2}$ are the first and second projections.

By the above remark, we may assume that $L$ is not big, so that $L^{g}=0$, where $g=\operatorname{dim} A$. By the Riemann-Roch theorem [loc. cit., Section 16], we have $\chi(L)=0$. Hence, it follows that $\operatorname{dim} K(L)>0$ by the vanishing theorem [loc. cit., Section 16].

Set $X:=K(L)_{\text {red. }}^{0}$ This is a subabelian variety of $A$. Thus, there exists a subabelian variety $Y \subset A$ such that the morphism $m: X \times Y \rightarrow A,(x, y) \mapsto x+y$ defined by the group law on $A$ is an isogeny [loc. cit., Section 19, Theorem 1]). Note that $\left.L\right|_{X} \in \operatorname{Pic}^{0}(X)$, because it is invariant under translations by any element of $X$ (see Remark 3.12).

First, we prove $m^{*} L \equiv p_{Y}^{*}\left(\left.L\right|_{Y}\right)$, where $p_{Y}: X \times Y \rightarrow Y$ is the second projection. By definition of $K(L)$, we get $m^{*} L=p_{X}^{*}\left(\left.L\right|_{X}\right)+p_{Y}^{*}\left(\left.L\right|_{Y}\right)$. Since $\left.L\right|_{X} \in \operatorname{Pic}^{0}(X)$, we have $\left.L\right|_{X} \equiv 0$, which proves $m^{*} L \equiv p_{Y}^{*}\left(\left.L\right|_{Y}\right)$.

Since $\operatorname{dim} Y<\operatorname{dim} A$, we may assume that $\left.L\right|_{Y}$ is numerically equivalent to a semiample line bundle by induction on $\operatorname{dim} A$. By Proposition 3.2, in order to complete the proof, it is sufficient to show that $p_{Y}^{*}\left(\left.L\right|_{Y}\right)$ descends to $A$. This is true, because $\mathrm{Pic}^{0}(A) \rightarrow \mathrm{Pic}^{0}(X \times Y)$ is surjective [loc. cit., Section 15, Theorem 1]).

Remark 3.12. Mumford [2008, Section 8] defines $\operatorname{Pic}^{0}(X)$, for an abelian variety $X$, to be the subgroup of $\operatorname{Pic}(X)$ consisting of line bundles invariant under translations by any element of $X$. The existence of the dual abelian variety and the Poincare line bundle [loc. cit., Section 13] shows that this definition is equivalent to the standard definition of $\operatorname{Pic}^{0}(X)$ as the identity component of the Picard functor.

Proposition 3.13. Let $X$ be a minimal ruled surface over an elliptic curve $B$. Then it follows that $\mathrm{NEF}(X) \subset \mathrm{NE}(X)$.

Proof. Let $C_{0} \subset X$ be a normalized section and $F$ a fiber of $X \rightarrow B$. Set $e:=-C_{0}^{2}$. When $e \geq 0$, we get

$$
\operatorname{NEF}(X)=\operatorname{Cone}\left(F, C_{0}+e F\right),
$$

and so nef line bundles are effective.

In what follows, we may assume $e=-1$ by [loc. cit., Chapter V, Theorem 2.15]. We know that

$$
\operatorname{NEF}(X)=\overline{\mathrm{NE}}(X)=\operatorname{Cone}\left(F, 2 C_{0}-F\right)
$$

by [loc. cit., Chapter V, Proposition 2.21]. Further, there exists a rank-two indecomposable vector bundle $E$ of degree one on $C$ such that $X \cong \mathbb{P}_{C}(E)$ holds. We denote the projection by $p: \mathbb{P}_{C}(E) \rightarrow C$. It is sufficient to show $H^{0}\left(X, O_{X}\left(2 C_{0}-p^{*} Q\right)\right) \neq$ 0 for some point $Q \in C$, because then $\overline{\mathrm{NE}}(X)=\mathrm{NE}(X)$. Note that

$$
H^{0}\left(X, \mathcal{O}_{X}\left(2 C_{0}-p^{*} Q\right)\right) \cong H^{0}\left(C, S^{2}(E) \otimes \mathcal{O}_{C}(-Q)\right)
$$


and $S^{2}(E)$ has both rank and degree equal to three (see [loc. cit., Chapter II, Example 5.16] and the proof of [loc. cit., Chapter V, Theorem 2.15]). When $S^{2}(E)$ is indecomposable, we can complete the proof by using the following proposition:

Proposition 3.14 [Atiyah 1957, Lemma 11]. Let $F$ be an indecomposable vector bundle of rank $r$ and degree $d$ on an elliptic curve. If $r=d$, then $F$ contains a degree-one line bundle as a subbundle.

When $S^{2}(E)$ is decomposable, it can be written as $S^{2}(E) \cong E_{1} \oplus E_{2}$, where $E_{1}$ is a line bundle and $E_{2}$ is a vector bundle of rank two. If $\operatorname{deg} E_{1} \geq 1$, then

$$
H^{0}\left(C, S^{2}(E) \otimes \mathcal{O}_{C}(-Q)\right) \supset H^{0}\left(C, E_{1} \otimes \mathcal{O}_{C}(-Q)\right) \neq 0
$$

for some point $Q \in C$, which finishes the proof in this case. If $\operatorname{deg} E_{1}<1$, then $\operatorname{deg} E_{2} \geq 3$, and so $\operatorname{deg}\left(E_{2} \otimes \mathcal{O}_{C}(-Q)\right) \geq 1$ for any point $Q \in C$. Therefore,

$$
H^{0}\left(C, S^{2}(E) \otimes \mathcal{O}_{C}(-Q)\right) \supset H^{0}\left(C, E_{2} \otimes \mathcal{O}_{C}(-Q)\right) \neq 0
$$

by the Riemann-Roch theorem.

\section{Reduction to surfaces}

The first step in the proof of Theorem 1.1 is to reduce the problem to the case of surfaces.

Theorem 4.1. Let $(X, \Delta)$ be a three-dimensional projective log pair defined over $\overline{\mathbb{F}}_{p}$, and $L$ a line bundle on $X$. If we assume that

- $L$ and $L-\left(K_{X}+\Delta\right)$ are nef and big,

- $\left.L\right|_{\operatorname{Supp}\lfloor\Delta\rfloor}$ is semiample,

then $L$ is semiample.

Here, we adopt the convention that, when $\lfloor\Delta\rfloor=0$, then $\left.L\right|_{\operatorname{Supp}\lfloor\Delta\rfloor}$ is automatically semiample.

Remark 4.2. Under the assumption $\lfloor\Delta\rfloor=0$, Theorem 4.1 was proved by Keel [1999, Theorem 0.5].

Proof of Theorem 1.1. Set $S:=\lfloor\Delta\rfloor$. Since $L$ is a big line bundle, we can decompose it as $L \sim_{\mathbb{Q}} A+E$, where $A$ is an ample and $E$ is an effective $\mathbb{Q}$-Cartier $\mathbb{Q}$-divisor. By Theorem 2.7, it is enough to show that $\left.L\right|_{E_{\text {red }}}$ is semiample.

We write $E_{\text {red }}=T+\sum_{i=1}^{m} E_{i}$, where $\operatorname{Supp}(T) \subset \operatorname{Supp}(S)$ and the $E_{i}$ are prime divisors not contained in $\operatorname{Supp}(S)$. Define $\lambda_{i} \in \mathbb{Q}$ so that $\Delta+\lambda_{i} E$ contains $E_{i}$ with coefficient one. Then, by definition of $\lambda_{i}$, there exists an effective $\mathbb{Q}$-divisor $\Gamma_{i}$ such that

$$
\Delta+\lambda_{i} E=E_{i}+\Gamma_{i}
$$


and $E_{i} \not \subset \operatorname{Supp}\left(\Gamma_{i}\right)$. Since $E_{i} \not \subset \operatorname{Supp}(S)$, it follows that $\lambda_{i}>0$. By rearranging indices, we may assume without loss of generality that

$$
\lambda_{1} \leq \lambda_{2} \leq \cdots \leq \lambda_{m},
$$

so we have

$$
T+\sum_{1 \leq j \leq i-1} E_{j} \leq \Gamma_{i}
$$

for each $i$.

We define $U_{0}:=\operatorname{Supp}(T)$ and $U_{i}:=U_{i-1} \cup E_{i}$ for $i>0$. Recall that it is sufficient to show that $L$ restricted to $U_{m}=\operatorname{Supp}\left(E_{\text {red }}\right)$ is semiample. We prove it by induction on $i$.

Observe that $\left.L\right|_{U_{0}}$ is semiample, because $U_{0}=\operatorname{Supp}(T) \subset \operatorname{Supp}(S)$ and $\left.L\right|_{S}$ is semiample by hypothesis. Let us assume that $\left.L\right|_{U_{i-1}}$ is semiample. In order to prove the semiampleness of $\left.L\right|_{U_{i}}$, we first prove the semiampleness of $\left.L\right|_{E_{i}}$.

We consider the normalization $p_{i}: \overline{E_{i}} \rightarrow E_{i}$. By adjunction (see Section 2C), there exists an effective $\mathbb{Q}$-divisor $\Delta_{\overline{E_{i}}}$ such that

$$
\left.\left(K_{X}+E_{i}+\Gamma_{i}\right)\right|_{\overline{E_{i}}} \sim K_{\overline{E_{i}}}+\Delta_{\overline{E_{i}}} .
$$

Lemma 4.3. $\left.L\right|_{\overline{E_{i}}}$ is semiample.

Proof. We define auxiliary divisors $D_{i}$ by

$$
D_{i}:=\left(1+\lambda_{i}\right) L-\left(K_{X}+\Delta+\lambda_{i} E\right) .
$$

Observe that

$$
D_{i}=L-\left(K_{X}+\Delta\right)+\lambda_{i}(L-E) \sim_{\mathbb{Q}}\left(L-\left(K_{X}+\Delta\right)\right)+\lambda_{i} A,
$$

and so $D_{i}$ is ample, because $L-\left(K_{X}+\Delta\right)$ is nef and $\lambda_{i} A$ is ample. Hence,

$$
\left.D_{i}\right|_{\overline{E_{i}}}=\left.\left(1+\lambda_{i}\right) L\right|_{\overline{E_{i}}}-\left(K_{\overline{E_{i}}}+\Delta_{\overline{E_{i}}}\right)
$$

is nef. Since $\left.\left(1+\lambda_{i}\right) L\right|_{\overline{E_{i}}}$ is also nef, the semiampleness of $\left.L\right|_{\overline{E_{i}}}$ follows from Theorem 1.4 and Remark 1.6.

Assume $\kappa\left(\left.L\right|_{\overline{E_{i}}}\right)$ is equal to 0 or 2 . Then the assumptions of Theorem 2.11 are satisfied, and so $\left.L\right|_{E_{i}}$ is semiample. Using Theorem 2.10 for $X_{1}=U_{i-1}$ and $X_{2}=E_{i}$, we get that $\left.L\right|_{U_{i}}$ is semiample.

In what follows, we assume $\kappa\left(\left.L\right|_{\overline{E_{i}}}\right)=1$.

Lemma 4.4. Let $\pi_{i}: \overline{E_{i}} \rightarrow Z_{i}$ be the map associated to the semiample line bundle $\left.L\right|_{\overline{E_{i}}}$, and let $F$ be a general fiber of $\pi_{i}$. Further, let $C_{i} \subset \overline{E_{i}}$ be the reduction of the conductor of the normalization $p_{i}: \overline{E_{i}} \rightarrow E_{i}$. Then $F$ and $C_{i}$ intersect in at most one point. 
Proof. Let $D_{i}$ be the $\mathbb{Q}$-divisor on $\overline{E_{i}}$ as in the proof of Lemma 4.3. Then, $D_{i}$ is ample, so we have $\left.F \cdot D_{i}\right|_{\overline{E_{i}}}>0$. Since $\left.F \cdot L\right|_{\overline{E_{i}}}=0$, we get

$$
F \cdot K_{\overline{E_{i}}}+F \cdot \Delta_{\overline{E_{i}}}<0 \text {. }
$$

Hence

$$
F \cdot \Delta_{\overline{E_{i}}}<-F \cdot K_{\overline{E_{i}}}=2-2 h^{1}\left(F, O_{F}\right) \leq 2 .
$$

By the adjunction formula (Proposition 2.2), the one-dimensional part of $C_{i}$ is contained in $\operatorname{Supp}\left(\left\lfloor\Delta_{\overline{E_{i}}}\right\rfloor\right)$. Hence, we get $\#\left(F \cap C_{i}\right) \leq F \cdot \Delta_{\overline{E_{i}}}<2$.

By this lemma, the assumptions of Theorem 2.11 are satisfied, and so $\left.L\right|_{E_{i}}$ is semiample. Let $\rho_{i}: E_{i} \rightarrow Z_{i}^{\prime}$ be the map associated to $\left.L\right|_{E_{i}}$, and let $G$ be a general fiber of $\rho_{i}$. Since $\pi_{i}$ is the Stein factorization of $\rho_{i} \circ p_{i}$, there exists a finite map $Z_{i} \rightarrow Z_{i}^{\prime}$ such that the following diagram commutes [Keel 1999, DefinitionLemma 1.0(4)]:

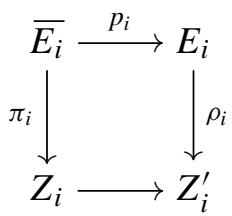

We want to apply Theorem 2.10 to $X_{1}=U_{i-1}$ and $X_{2}=E_{i}$ to show that $\left.L\right|_{U_{i}}$ is semiample. It is sufficient to prove that $G$ intersects $U_{i-1} \cap E_{i}$ in at most one point.

Recall that

$$
T+\sum_{1 \leq j \leq i-1} E_{j} \leq \Gamma_{i}, \quad U_{i-1}=\operatorname{Supp}\left(T+\sum_{1 \leq j \leq i-1} E_{j}\right) .
$$

Hence, the one-dimensional part of $p_{i}^{-1}\left(U_{i-1} \cap E_{i}\right)$ is contained in $\operatorname{Supp}\left(\left\lfloor\Delta_{\overline{E_{i}}}\right\rfloor\right)$ by the adjunction formula (Proposition 2.2). By the proof of Lemma 4.4, we can conclude

$$
\begin{aligned}
\#\left(\left(U_{i-1} \cap E_{i}\right) \cap G\right) & =\#\left(p_{i}\left(p_{i}^{-1}\left(U_{i-1} \cap E_{i}\right) \cap F\right)\right) \\
& \leq \#\left(p_{i}^{-1}\left(U_{i-1} \cap E_{i}\right) \cap F\right) \\
& \leq F \cdot \Delta_{\overline{E_{i}}}<2,
\end{aligned}
$$

which completes the proof.

\section{Semiampleness on nonirreducible surfaces}

In this section, we prove Theorem 5.2. Before stating it, we need to introduce some notation. Let $S$ be a pure two-dimensional reduced projective scheme over $\overline{\mathbb{F}}_{p}$, and let $S=\bigcup_{i=1}^{n} S_{i}$ be its irreducible decomposition and $\bar{S} \rightarrow S$ its normalization. Let $\mathscr{D} \subset S$ and $\mathscr{C} \subset \bar{S}$ be the conductors of $S$. Let $\bar{C} \stackrel{\text { norm. }}{\longrightarrow} \mathscr{C}_{\text {red }} \longrightarrow \mathscr{C}$ and $\bar{D} \stackrel{\text { norm. }}{\longrightarrow} \mathscr{D}$ red $\longrightarrow \mathscr{D}$ 
be the compositions of the reduction map and the normalization. Then we have a natural morphism $f: \bar{C} \rightarrow \bar{D}$ such that the following diagram commutes:

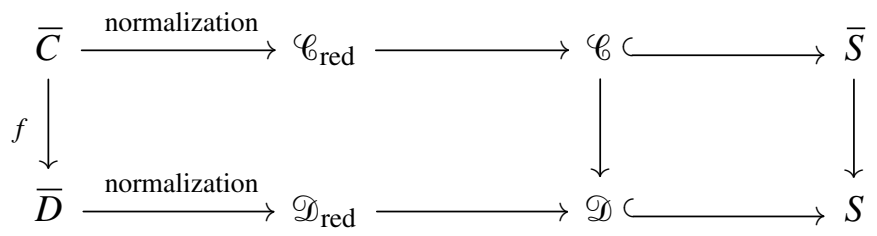

Consider the one-dimensional part $\bar{C}^{(1)}$ of $\bar{C}$ and the restriction $f: \bar{C}^{(1)} \rightarrow \bar{D}$. We say that $S$ satisfies the condition ( $\star$ ) when the restriction of $f$ to any one-dimensional connected component of $\bar{C}$ is an isomorphism onto its image. Further, we say that $S$ satisfies the condition $(\star \star)$ when any fiber of the restriction $f: \bar{C}^{(1)} \rightarrow \bar{D}$ has length at most two.

Remark 5.1. If each $S_{i}$ is normal, then $S$ satisfies the condition ( $\star$ ). If $S$ is regular or nodal in codimension one, then $S$ satisfies the condition $(\star \star)$. See the proof of Theorem 1.1.

Theorem 5.2. Let $S$ be a pure two-dimensional reduced projective scheme over $\overline{\mathbb{F}}_{p}$, and let $S=\bigcup_{i=1}^{n} S_{i}$ be its irreducible decomposition. Let $L$ be a nef line bundle on $S$. Suppose that $S$ satisfies the condition $(\star)$ or $(\star \star)$ defined above and that there exists an effective $\mathbb{Q}$-divisor $\Delta_{\bar{S}}$ on the normalization $\bar{S}$ of $S$ such that:

- $\left.L\right|_{\bar{S}}-\left(K_{\bar{S}}+\Delta_{\bar{S}}\right)$ is nef.

- $\operatorname{Supp}\left(\mathscr{C}^{(1)}\right)$ is contained in $\operatorname{Supp}\left(\left\lfloor\Delta_{\bar{S}}\right\rfloor\right)$, where $\mathscr{C}^{(1)} \subset \bar{S}$ is the one-dimensional part of the conductor scheme of the normalization of $S$.

Then L is semiample.

Proof. We use the same notation as above. Let $v: \bar{S}:=\bigsqcup \overline{S_{i}} \rightarrow S$ be the normalization of $S$. Set $\Delta_{\overline{S_{i}}}:=\left.\Delta_{\bar{S}}\right|_{\overline{S_{i}}}$. We know that the $\left.L\right|_{\overline{S_{i}}}$ are semiample from Theorem 1.4. Let $g_{i}: \overline{S_{i}} \rightarrow Z_{i}$ be the map associated to $\left.L\right|_{\overline{S_{i}}}$. Set $g: \bar{S} \rightarrow Z$, where $g:=\bigsqcup g_{i}$ and $Z:=\bigsqcup Z_{i}$. If $\operatorname{dim} Z_{i} \neq 1$, then $g_{i}$ satisfies the conditions of Theorem 2.10. Hence, we may assume that $\operatorname{dim} Z_{i}=1$ for any $i$ by the inductive argument in the proof of Theorem 4.1.

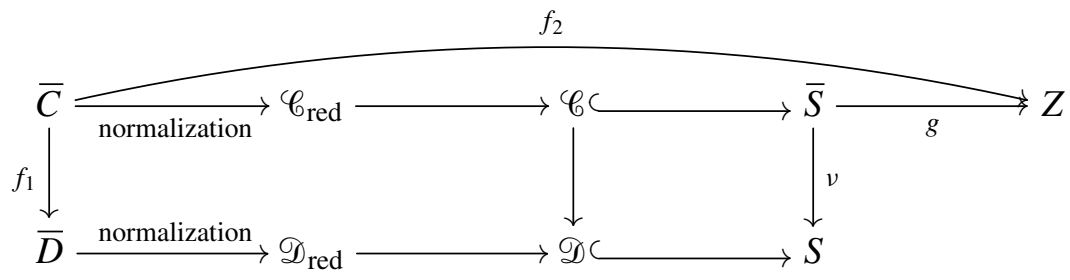


By Remark 2.1, it is sufficient to show that, for any point $p \in \bar{S}$, there exist $m \geq 1$ and a section $s \in H^{0}\left(\bar{S},\left.L^{\otimes m}\right|_{\bar{S}}\right)$ such that $\left.s\right|_{\mathscr{C}}$ descends to $\mathscr{D}$ and $\left.s\right|_{p} \neq 0$. To obtain this, we prove the following claim:

Claim 5.3. For any finite set $F \subset \bar{S}$ of closed points of $\bar{S}$, we can find $m \geq 1$ and $a$ section $s \in H^{0}\left(\bar{S},\left.L^{\otimes m}\right|_{\bar{S}}\right)$ such that $\left.s\right|_{\bar{C}}$ descends to $\bar{D}$ and $s$ is nowhere-vanishing on $F$.

First, we assume this claim and complete the proof of Theorem 5.2. Let $F^{\prime} \subset \mathscr{D}_{\text {red }}$ be the conductor corresponding to the normalization $\bar{D} \rightarrow \mathscr{D}_{\text {red }}$. Let $F^{\prime \prime}$ be the image of $F^{\prime}$ in $S$. Set $F:=v^{-1}\left(F^{\prime \prime}\right) \cup\{p\}$. Then $F$ is a finite set.

By Claim 5.3, we can take $s \in H^{0}\left(\bar{S},\left.L^{\otimes m}\right|_{\bar{S}}\right)$ and $s_{\bar{D}} \in H^{0}\left(\bar{D},\left.L^{\otimes m}\right|_{\bar{D}}\right)$ such that $\left.s\right|_{\bar{C}}=\left.s_{\bar{D}}\right|_{\bar{C}}$ and $s$ is nowhere-vanishing on $F$. By Lemma 2.5 , if we replace $s_{\bar{D}}$ by some power of it, then $s_{\bar{D}}$ descends to a section $s_{\mathscr{D}_{\text {red }}}$ on $\mathscr{D}_{\text {red }}$. Since $\mathscr{D}_{\text {red }} \rightarrow \mathscr{D}$ is a universal homeomorphism, $s_{\mathscr{D}_{\text {red }}}$ descends to a section $s_{\mathscr{D}}$ on $\mathscr{D}$, if we replace $s_{\bar{D}}$ by some power of it (see Theorem 2.9).

It is sufficient to show that $\left.s\right|_{\mathscr{C}}=\left.s_{\mathscr{D}}\right|_{\mathscr{C}}$. By construction, $\left.\left(\left.s\right|_{\mathscr{C}}\right)\right|_{\bar{C}}=\left.\left(\left.s_{\mathscr{D}}\right|_{\mathscr{C}}\right)\right|_{\bar{C}}$ holds. Since $\bar{C} \rightarrow \mathscr{C}_{\text {red }}$ is surjective, we get $\left.\left(\left.s\right|_{\mathscr{C}}\right)\right|_{\mathscr{C}_{\text {red }}}=\left.\left(\left.s_{\mathscr{D}}\right|_{\mathscr{C}}\right)\right|_{\mathscr{C}_{\text {red }}}$. As $\mathscr{C}_{\text {red }} \rightarrow \mathscr{C}$ is a universal homeomorphism, if we replace $s$ by some power of it, then we get $\left.s\right|_{\mathscr{C}}=\left.s_{\mathscr{D}}\right|_{\mathscr{C}}$ (see Theorem 2.9). This completes the proof of Theorem 5.2.

Proof of Claim 5.3. Let $f_{1}$ and $f_{2}$ be as in the above diagram. For a one-dimensional scheme $X$, we write $X=X^{(0)} \sqcup X^{(1)}$, where $X^{(i)}$ is the $i$-dimensional part. Further, we write $\bar{C}^{(1)}=\bar{C}^{\mathrm{h}} \sqcup \bar{C}^{\mathrm{v}}$, where $\bar{C}^{\mathrm{h}}$ is the $f_{2}$-horizontal part and $\bar{C}^{\mathrm{v}}$ is the $f_{2}$-vertical part.

First, we claim that, for any closed point $p \in Z$, the inverse image of $p$ by $\bar{C}^{\mathrm{h}} \rightarrow Z$ has length at most two. This can be proved as follows: by the nefness of $L-\left(K_{\overline{S_{i}}}+\Delta_{\overline{S_{i}}}\right)$, we have

$$
0 \leq G_{i} \cdot\left(L-\left(K_{\overline{S_{i}}}+\Delta_{\overline{S_{i}}}\right)\right)=-G_{i} \cdot\left(K_{\overline{S_{i}}}+\Delta_{\overline{S_{i}}}\right) \leq 2-G_{i} \cdot \Delta_{\overline{S_{i}}},
$$

where $G_{i}$ is a general fiber of $g_{i}: \overline{S_{i}} \rightarrow Z_{i}$. Since the one-dimensional part of $\left.\mathscr{C}\right|_{\overline{S_{i}}}$ is contained in $\operatorname{Supp}\left(\left\lfloor\Delta_{\overline{S_{i}}}\right\rfloor\right)$, we have

$$
\#\left(\left.G_{i} \cap \mathscr{C}\right|_{\overline{S_{i}}}\right) \leq G_{i} \cdot \Delta_{\overline{S_{i}}} \leq 2 .
$$

Hence, $f_{2}: \bar{C}^{\mathrm{h}} \rightarrow Z$ satisfies the assumption of Lemma 5.4. Further, by conditions $(\star)$ and $(\star \star), f_{1}: \bar{C}^{\mathrm{h}} \rightarrow D^{\prime}$ also satisfies the assumption of Lemma 5.4, where we define $D^{\prime}:=f_{1}\left(\bar{C}^{\mathrm{h}}\right)$.
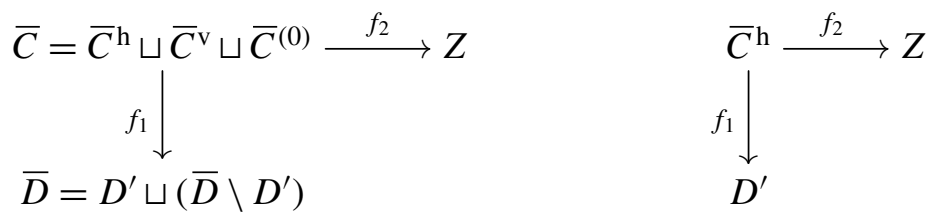
By Lemma 5.4, we can find sections $s_{\bar{D}} \in H^{0}\left(\bar{D},\left.L^{\otimes m}\right|_{\bar{D}}\right)$ and $s_{Z} \in H^{0}\left(Z,\left.L^{\otimes m}\right|_{Z}\right)$ such that $\left.s_{\bar{D}}\right|_{\bar{C}^{\mathrm{h}}}=\left.s_{Z}\right|_{\bar{C}^{\mathrm{h}}}$ holds, the section $s_{Z}$ is nowhere-vanishing on the finite set $g(F) \cup f_{2}\left(\bar{C}^{\mathrm{v}} \sqcup \bar{C}^{(0)}\right)$, and the section $s_{\bar{D}}$ is nowhere-vanishing on $\bar{D} \backslash D^{\prime}$. Since $\left.L\right|_{\bar{C}^{\mathrm{v}} \sqcup \bar{C}^{(0)}}$ is trivial, we have $\left.s \frac{n}{\bar{D}}\right|_{\bar{C}^{\mathrm{v}} \sqcup \bar{C}^{(0)}}=\left.s_{Z}^{n}\right|_{\bar{C}^{\mathrm{v}} \sqcup \bar{C}^{(0)}}$ for some $n \geq 1$ by Lemma 2.4 . Therefore, we get $\left.s_{\bar{D}}^{n}\right|_{\bar{C}}=\left.s_{Z}^{n}\right|_{\bar{C}}$ and this completes the proof of Claim 5.3.

Finally, we show the next lemma, which was used in the proof of Theorem 5.2.

Lemma 5.4. Let $X, Z_{1}, Z_{2}$ be disjoint unions of smooth proper curves and $f_{1}: X \rightarrow$ $Z_{1}, f_{2}: X \rightarrow Z_{2}$ finite surjective morphisms. Let $L_{1}$ and $L_{2}$ be line bundles on $Z_{1}$ and $Z_{2}$, respectively, such that $f_{1}^{*} L_{1}=f_{2}^{*} L_{2}$. Suppose that $L:=f_{1}^{*} L_{1}=f_{2}^{*} L_{2}$ is semiample. Further, assume that each $f_{i}$ satisfies either of the following conditions:

- The restriction of $f_{i}$ to any connected component of $X$ is an isomorphism onto its image.

- Any fiber of $f_{i}$ has length at most two.

Then, for any finite set $F \subset X$ of closed points of $X$, we can take $m \geq 1$ and $a$ section $s \in H^{0}\left(X, L^{\otimes m}\right)$ such that $s$ is nowhere-vanishing on $F$ and $s$ descends to both $Z_{1}$ and $Z_{2}$.

Proof. First, we prove that there exists a finite group $G_{i}$ acting on $X$ such that $X \rightarrow Z_{i}$ decomposes into the quotient morphism $X \rightarrow X / G_{i}$ and a universal homeomorphism $X / G_{i} \rightarrow Z_{i}$ :

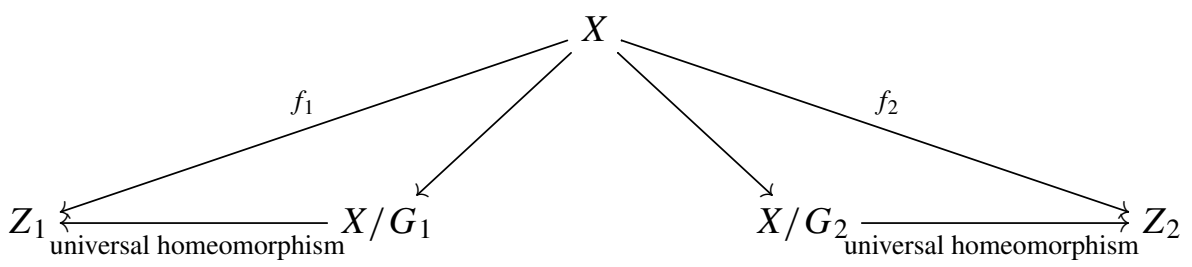

This is trivial when the restriction of $f_{i}$ to any connected component of $X$ is an isomorphism. Indeed, it is sufficient to take $G_{i}$ such that it identifies the components with the same image under $f_{i}$. Then $X \rightarrow Z_{i}$ is isomorphic to the quotient morphism $X \rightarrow X / G_{i}$.

For the second case, assume that any fiber of $f_{i}$ has length at most two. Let $Z_{i}^{\prime}$ be a connected component of $Z_{i}$. Set $X^{\prime}=f_{i}^{-1}\left(Z_{i}^{\prime}\right)$. There are four possibilities:

(1) $X^{\prime}$ is connected and $X^{\prime} \rightarrow Z_{i}^{\prime}$ is an isomorphism.

(2) $X^{\prime}$ is connected and $X^{\prime} \rightarrow Z_{i}^{\prime}$ is the Frobenius map (this case may only occur for characteristic $p=2$ ).

(3) $X^{\prime}$ is connected and every fiber of $X^{\prime} \rightarrow Z_{i}^{\prime}$ has length two. There exists an involution $\iota: X^{\prime} \rightarrow X^{\prime}$ such that $X^{\prime} \rightarrow Z_{i}^{\prime}$ is the quotient by $\iota$. 
(4) $X^{\prime}$ has two connected components $X_{1}^{\prime}$ and $X_{2}^{\prime}$. Further, $X_{1}^{\prime} \rightarrow Z_{i}^{\prime}$ and $X_{2}^{\prime} \rightarrow Z_{i}^{\prime}$ are isomorphisms. In this case, we have $X_{1}^{\prime} \cong X_{2}^{\prime}$.

In the cases (3) and (4), we have a finite group $G^{\prime}$ acting on $X^{\prime}$ such that the morphism $X^{\prime} \rightarrow Z_{i}^{\prime}$ is isomorphic to the quotient morphism $X^{\prime} \rightarrow X^{\prime} / G^{\prime}$.

Hence, we have a finite group $G_{i}$ acting on $X$ such that the morphism $X \rightarrow Z_{i}$ decomposes as $X \rightarrow X / G_{i} \rightarrow Z_{i}$, where $X \rightarrow X / G_{i}$ is the quotient morphism and $X / G_{i} \rightarrow Z_{i}$ is a universal homeomorphism (actually, if we restrict it to a connected component, it is either an isomorphism or the Frobenius map).

Note that $L=g^{*} L$ for any $g \in G_{i}$. We claim that if $s \in H^{0}\left(X, L^{\otimes m}\right)$ is $G_{i^{-}}$ equivariant, then $s^{p^{e}}$ descends to $Z_{i}$ for sufficiently large $e$. This is because $s$ descends to $X / G_{i}$ and $X / G_{i} \rightarrow Z_{i}$ is a universal homeomorphism (see Theorem 2.9).

Let $G:=G_{1} G_{2} \subset \operatorname{Aut}(X)$ be a composition of the groups, and let $S \subset X$ be the $G$-orbit of the set $F$. By Lemma 2.6, $G$ is a finite group, and therefore $S$ is a finite set.

Take $m \geq 1$ and a section $s \in H^{0}\left(X, L^{\otimes m}\right)$ such that $s$ is nowhere-vanishing on $S$. Set

$$
s^{G}:=\prod_{\sigma \in G} \sigma^{*} s \in H^{0}\left(X, L^{\otimes m|G|}\right) .
$$

The section $s^{G}$ is $G_{i}$-invariant for each $i$ and nowhere-vanishing on $F$. Hence, $\left(s^{G}\right)^{p^{e}}$ satisfies the statement of the lemma for sufficiently large $e \geq 1$.

The main issue of this section is related to the following question, discussed by Keel [2003].

Question 5.5. Let $L$ be a line bundle on a variety $X$ and let $p: \bar{X} \rightarrow X$ be the normalization of $X$. Assume that $p^{*} L$ is semiample. What additional assumptions are necessary for $L$ to be semiample?

\section{Proof of Theorem 1.1}

In this section, we prove Theorem 1.1 using Theorem 4.1 and Theorem 5.2.

Proof of Theorem 1.1. Let $S:=\lfloor\Delta\rfloor$. By Theorem 4.1, it is sufficient to show that $\left.L\right|_{\text {Supp (S) }}$ is semiample. Note that in both case (1) and case (2), all the coefficients of $\Delta$ are at most one.

By the adjunction formula (Proposition 2.2), if we define $\Delta_{\bar{S}}$ on $\bar{S}$ so that $\left.\left(K_{X}+\Delta\right)\right|_{\bar{S}}=K_{\bar{S}}+\Delta_{\bar{S}}$, then $\Delta_{\bar{S}}$ satisfies the conditions in the statement of Theorem 5.2.

In the case (2), that is, the case when each component $S_{i}$ of $S$ is normal, $S$ clearly satisfies the condition $(\star)$. In the case $(1)$, that is, the case when $(X, \Delta)$ is $\log$ canonical, the surface $S$ is regular or nodal in codimension one (see [Kollár 2013, Corollary 2.32]), and so $S$ satisfies condition ( $\star$ ) (see [Kollár 2013, Claim 1.41.1] or [Tanaka 2014, Lemma 3.4, 3.5]). 
Thus, we can complete the proof by using Theorem 5.2.

We easily deduce Corollary 1.2:

Proof of Corollary 1.2. It is enough to take $L=2\left(K_{X}+\Delta\right)$ and $L=-\left(K_{X}+\Delta\right)$, respectively.

\section{Examples}

Theorem 1.1 does not hold if we do not impose any conditions on $\Delta$. It is in fact possible to construct a nef and big line bundle $L$ on a smooth threefold $X$ such that $L-\left(K_{X}+\Delta\right)$ is nef and big for $\Delta \geq 0$, but $L$ is not semiample. We construct such $L$ and $\Delta$ in the following way:

Example 7.1. Let $L$ be a nef and big line bundle on a smooth threefold which is not semiample (see an example in [Totaro 2009, Theorem 7.1]). Since $L$ is big, we can write $L=A+E$ for an ample $\mathbb{Q}$-Cartier $\mathbb{Q}$-divisor $A$ and an effective $\mathbb{Q}$-Cartier $\mathbb{Q}$-divisor $E$. Take $\Delta=m E$ for $m \in \mathbb{N}$ big enough. Then $m L-\left(K_{X}+\Delta\right)$ is an ample Cartier divisor, and so the pair $L^{\prime}:=m L$ and $\Delta$ is an example which we were looking for.

Theorem 1.1 does not hold over algebraically closed fields $k \neq \overline{\mathbb{F}}_{p}$ even in the two-dimensional case:

Example 7.2 [Tanaka 2012, Example 19.3]. Let $C_{0} \subset \mathbb{P}^{2}$ be an elliptic curve in $\mathbb{P}^{2}$, and let $p_{1}, \ldots, p_{10} \in C_{0}$ be ten general points on $C_{0}$. Let $X$ be the blowup of $\mathbb{P}^{2}$ along these ten points, and $C$ the proper transform of $C_{0}$. Note that $K_{X}+C \sim 0$ and $C^{2}=-1$.

Take an ample divisor $H$ on $X$, and set $L:=H+a C$, where $a:=H \cdot C>0$. Note that $L$ is a nef and big divisor. Further, $(X, C)$ is $\log$ canonical, and $L-\left(K_{X}+C\right)$ is also nef and big. Nevertheless, $L$ is not semiample if the base field is not $\overline{\mathbb{F}}_{p}$. This is because $L \cdot C=0$, but the elliptic curve $C$ is not contractible.

Corollary 1.2(2) also does not hold over algebraically closed fields $k \neq \overline{\mathbb{F}}_{p}$ :

Example 7.3 [Gongyo 2012, Example 5.2]. Let $S$ be the blowup of $\mathbb{P}^{2}$ along nine general points. Note that $-K_{S}$ is nef but not semiample if the base field is not $\overline{\mathbb{F}}_{p}$. Take a very ample divisor $H$ on $S$, and set $X:=\mathbb{P}_{S}\left(\mathcal{O}_{S} \oplus \mathcal{O}_{S}(-H)\right)$. Let $E$ be the tautological section of $O_{S} \oplus \mathcal{O}_{S}(-H)$. Since $E \cong S$, it follows that $-K_{E}$ is not semiample.

Then, $(X, E)$ is $\log$ canonical, and $L:=-\left(K_{X}+E\right)$ is nef and big by the nefness of $-K_{S}$ (for details, see [Gongyo 2012, Example 5.2]). Nevertheless, $L$ is not semiample, because $\left.L\right|_{E}=-K_{E}$ is not semiample. 


\section{Acknowledgements}

We would like to thank Paolo Cascini and Yoshinori Gongyo for suggesting this problem and for their constant support and guidance.

The project started during the Pragmatic Research Summer School 2013 in Catania and part of the work carried on during the visit of Martinelli and Witaszek to the University of Tokyo. We are grateful to the University of Catania and the organizers of the Pragmatic Research School, as well as to Yujiro Kawamata and Yoshinori Gongyo for the invitation to the University of Tokyo.

We are grateful to Andrea Fanelli, Enrica Floris, Atsushi Ito, and Hiromu Tanaka for useful comments and suggestions.

Martinelli is supported by a Roth studentship. Nakamura is supported by the Grant-in-Aid for Scientific Research (KAKENHI No. 25-3003) and the Program for Leading Graduate Schools, MEXT, Japan. Witaszek was supported by Bonn International Graduate School's Pre-Ph.D. scholarship.

\section{References}

[Artin 1962] M. Artin, "Some numerical criteria for contractability of curves on algebraic surfaces", Amer. J. Math. 84 (1962), 485-496. MR 26 \#3704 Zbl 0105.14404

[Atiyah 1957] M. F. Atiyah, "Vector bundles over an elliptic curve", Proc. London Math. Soc. (3) 7 (1957), 414-452. MR 24 \#A1274 Zbl 0084.17305

[Birkar 2013] C. Birkar, "Existence of flips and minimal models for 3-folds in char p", preprint, 2013. arXiv 1311.3098v1

[Bombieri and Mumford 1976] E. Bombieri and D. Mumford, "Enriques' classification of surfaces in char p, III”, Invent. Math. 35 (1976), 197-232. MR 58 \#10922b Zbl 0336.14010

[Bombieri and Mumford 1977] E. Bombieri and D. Mumford, "Enriques' classification of surfaces in char p, II", pp. 23-42 in Complex analysis and algebraic geometry, edited by W. L. Baily, Jr., Iwanami Shoten, Tokyo, 1977. MR 58 \#10922a Zbl 0348.14021

[Cascini et al. 2014] P. Cascini, J. McKernan, and M. Mustață, "The augmented base locus in positive characteristic”, Proc. Edinb. Math. Soc. (2) 57:1 (2014), 79-87. MR 3165013 Zbl 1290.14006

[Fong and $\mathrm{M}^{\mathrm{C}}$ Kernan 1992] L.-Y. Fong and J. $\mathrm{M}^{\mathrm{c}}$ Kernan, "Log abundance for surfaces", pp. 127-137 in Flips and abundance for algebraic threefolds (Salt Lake City, 1991), edited by J. Kollár, Astérisque 211, Société Mathématique de France, Paris, 1992. MR 94f:14013 Zbl 0807.14029

[Fujino 2000] O. Fujino, "Abundance theorem for semi log canonical threefolds", Duke Math. J. 102:3 (2000), 513-532. MR 2001c:14032 Zbl 0986.14007

[Gongyo 2012] Y. Gongyo, "On weak Fano varieties with log canonical singularities", J. Reine Angew. Math. 665 (2012), 237-252. MR 2908745 Zbl 1243.14018

[Hartshorne 1977] R. Hartshorne, Algebraic geometry, Graduate Texts in Mathematics 52, Springer, New York-Heidelberg, 1977. MR 57 \#3116 Zbl 0367.14001

[Keel 1999] S. Keel, "Basepoint freeness for nef and big line bundles in positive characteristic", Ann. of Math. (2) 149:1 (1999), 253-286. MR 2000j:14011 Zbl 0954.14004

[Keel 2003] S. Keel, "Polarized pushouts over finite fields", Comm. Algebra 31:8 (2003), 3955-3982. MR 2004h:14010 Zbl 1051.14017 
[Kollár 2013] J. Kollár, Singularities of the minimal model program, Cambridge Tracts in Mathematics 200, Cambridge University Press, 2013. MR 3057950 Zbl 1282.14028

[Kollár and Mori 1998] J. Kollár and S. Mori, Birational geometry of algebraic varieties, Cambridge Tracts in Mathematics 134, Cambridge University Press, 1998. MR 2000b:14018 Zbl 0926.14003

[Lehmann 2012] B. Lehmann, "Numerical triviality and pullbacks", preprint, 2012. arXiv 1109. $4382 \mathrm{v} 3$

[Liedtke 2013] C. Liedtke, "Algebraic surfaces in positive characteristic", pp. 229-292 in Birational geometry, rational curves, and arithmetic, edited by F. Bogomolov et al., Springer, New York, 2013. MR 3114931 Zbl 06211443

[Liu 2002] Q. Liu, Algebraic geometry and arithmetic curves, Oxford Graduate Texts in Mathematics 6, Oxford University Press, 2002. MR 2003g:14001 Zbl 0996.14005

[Mumford 1969] D. Mumford, "Enriques' classification of surfaces in char p, I”, pp. 325-339 in Global Analysis (Papers in Honor of K. Kodaira), edited by D. C. Spencer and S. Iyanaga, University of Tokyo Press, 1969. MR 40 \#7266 Zbl 0188.53201

[Mumford 2008] D. Mumford, Abelian varieties, Tata Institute of Fundamental Research Studies in Mathematics 5, Hindustan Book Agency, New Delhi, 2008. Corrected reprint of the 1974 edition. MR 2010e:14040 Zbl 1177.14001

[Silverman 2009] J. H. Silverman, The arithmetic of elliptic curves, 2nd ed., Graduate Texts in Mathematics 106, Springer, Dordrecht, 2009. MR 2010i:11005 Zbl 1194.11005

[Tanaka 2012] H. Tanaka, "Minimal models and abundance for positive characteristic log surfaces", preprint, 2012. arXiv 1201.5699v2

[Tanaka 2014] H. Tanaka, "Abundance theorem for semi log canonical surfaces in positive characteristic", preprint, 2014. arXiv 1301.6889v2

[Totaro 2009] B. Totaro, "Moving codimension-one subvarieties over finite fields", Amer. J. Math. 131:6 (2009), 1815-1833. MR 2011c:14069 Zbl 1200.14022

[Xu 2013] C. Xu, "On base point free theorem of threefolds in positive characteristic", preprint, 2013. arXiv $1311.3819 \mathrm{v} 1$

Communicated by Shigefumi Mori

Received 2014-09-02 Revised 2015-01-11 Accepted 2015-02-16

d.martinelli12@imperial.ac.uk Department of Mathematics, Imperial College London, 180 Queen's Gate, London SW7 2AZ, United Kingdom

nakamura@ms.u-tokyo.ac.jp Graduate School of Mathematical Sciences, The University of Tokyo, 3-8-1 Komaba, Meguro-ku, Tokyo 153-8914, Japan

jakub.witaszek.14@ucl.ac.uk The London School of Geometry and Number Theory, Department of Mathematics, University College London, Gower Street, London WC1E 6BT, United Kingdom 


\section{Algebra \& Number Theory}

msp.org/ant

\section{EDITORS}

MANAGING EDITOR

Bjorn Poonen

Massachusetts Institute of Technology

Cambridge, USA

\author{
EDITORIAL BOARD CHAIR \\ David Eisenbud \\ University of California \\ Berkeley, USA
}

\section{BOARD OF EDITORS}

Georgia Benkart

Dave Benson

Richard E. Borcherds

John H. Coates

J-L. Colliot-Thélène

Brian D. Conrad

Hélène Esnault

Hubert Flenner

Edward Frenkel

Andrew Granville

Joseph Gubeladze

Roger Heath-Brown

Craig Huneke

János Kollár

Yuri Manin

Barry Mazur

Philippe Michel
University of Wisconsin, Madison, USA

University of Aberdeen, Scotland

University of California, Berkeley, USA

University of Cambridge, UK

CNRS, Université Paris-Sud, France

University of Michigan, USA

Freie Universität Berlin, Germany

Ruhr-Universität, Germany

University of California, Berkeley, USA

Université de Montréal, Canada

San Francisco State University, USA

Oxford University, UK

University of Virginia, USA

Princeton University, USA

Northwestern University, USA

Harvard University, USA

École Polytechnique Fédérale de Lausanne
Susan Montgomery

Shigefumi Mori

Raman Parimala

Jonathan Pila

Anand Pillay

Victor Reiner

Peter Sarnak

Joseph H. Silverman

Michael Singer

Vasudevan Srinivas

J. Toby Stafford

Ravi Vakil

Michel van den Bergh

Marie-France Vignéras

Kei-Ichi Watanabe

Efim Zelmanov

Shou-Wu Zhang
University of Southern California, USA

RIMS, Kyoto University, Japan

Emory University, USA

University of Oxford, UK

University of Notre Dame, USA

University of Minnesota, USA

Princeton University, USA

Brown University, USA

North Carolina State University, USA

Tata Inst. of Fund. Research, India

University of Michigan, USA

Stanford University, USA

Hasselt University, Belgium

Université Paris VII, France

Nihon University, Japan

University of California, San Diego, USA

Princeton University, USA

\section{PRODUCTION}

production@msp.org

Silvio Levy, Scientific Editor

See inside back cover or msp.org/ant for submission instructions.

The subscription price for 2015 is US $\$ 255 /$ year for the electronic version, and $\$ 440 /$ year ( $+\$ 55$, if shipping outside the US) for print and electronic. Subscriptions, requests for back issues and changes of subscribers address should be sent to MSP.

Algebra \& Number Theory (ISSN 1944-7833 electronic, 1937-0652 printed) at Mathematical Sciences Publishers, 798 Evans Hall \#3840, c/o University of California, Berkeley, CA 94720-3840 is published continuously online. Periodical rate postage paid at Berkeley, CA 94704, and additional mailing offices.

ANT peer review and production are managed by EditFLOW ${ }^{\circledR}$ from MSP.

\section{PUBLISHED BY}

- mathematical sciences publishers

nonprofit scientific publishing

http://msp.org/

(C) 2015 Mathematical Sciences Publishers 


\section{Algebra \& Number Theory}

Volume $9 \quad$ No. $3 \quad 2015$

Hurwitz monodromy and full number fields

DAVID P. ROBERTS and AKSHAY VENKATESH

The characteristic polynomial of the Adams operators on graded connected Hopf algebras

Marcelo Aguiar and Aaron Lauve

Secant spaces and syzygies of special line bundles on curves

MARIAN APRODU and EDOARDO SERNESI

Complex group algebras of the double covers of the symmetric and alternating groups

601

Christine Bessenrodt, Hung Ngoc Nguyen, Jørn B. Olsson and Hung P. TONG-VIET

Fano schemes of determinants and permanents

Melody Chan and NATHan Ilten

Triple intersection formulas for isotropic Grassmannians

VIJAY RAVIKUMAR

On the basepoint-free theorem for log canonical threefolds over the algebraic closure of a finite field

Diletta Martinelli, Yusuke NaKamura and Jakub Witaszek

The torsion group of endotrivial modules

JON F. CARLSON and JACQUES THÉVENAZ 